\title{
Management of Severe Traumatic Brain Injury in Children by Decompressive Craniectomy: Technical Note and Therapy Concepts
}

\author{
Hejazi N, MD* and Al-Kassab M, MD \\ Department of Neurosurgery, Bad Hersfeld, Germany \\ *Corresponding author: Hejazi N, Department of Neurosurgery, Bad Hersfeld, Germany
}

\begin{abstract}
The goal of this study was to evaluate the therapeutic role of decompressive craniectomy for severe brain injury in children. Between 1993 and 2020 we treated 37 children with severe brain injury (Glasgow Coma Scores lower than 8) and primary intracranial pressure of $30 \mathrm{mmHg}$ or higher. In all cases follow up data could be obtained. Mean age was 6.5 years (range 2 years - 16 years). Initial computerized tomography scans of all patients demonstrated diffuse brain injury with generalized brain swelling without large mass lesion. In all patients unilateral decompressive craniectomy was performed. Three patients died during the first $48 \mathrm{~h}$ post emergency operation, all the other are still alive with the following Glasgow Outcome Scores: good recovery (27), moderate disability (5), and vegetative state (2). There were no major complications. In 4 cases there was an enlargement of pre-existing contusions without a significant mass effect. The burst- suppression pattern was always maintained for at least one week, with a median duration of 10 days. A significant benefit could be demonstrated after unilateral decompressive craniectomy in the young patient population, which seems to have a more positive influence on outcome and survival when performed immediately.
\end{abstract}

\section{Keywords}

Decompressive craniectomy, Severe brain injury, Raised intracranial pressure, Children, Outcome, Cerebral oedema, Cerebral perfusion pressure

\section{Abbreviations}

CPP: Cerebral Perfusion Pressure; CT: Computerized Tomography; MRI: Magnetic Resonance Tomography; GCS: Glasgow Coma Score; GOS: Glasgow Outcome Score; ICP: Intracranial Pressure; TBI: Traumatic Brain Injury; UDC: Unilateral Decompressive Craniectomy

\section{Introduction}

Traumatic brain injury (TBI) is the most common cause of mortality in children younger than 16 years of age. It is twice as common as cancer and congenital diseases combined [1-6]. Head injury is present in $75 \%$ of children with trauma and $70 \%$ of all traumatic deaths are due to the TBI [7-11]. Most instances of death occur in the severe brain-injured child with a Glasgow Coma Score (GCS) of 8 or less $[2,7,8,12,13]$. Because of the frequency of this condition in the paediatric population, it is important that it be handled appropriately and effectively. Recommendations have been made by the Brain Trauma Foundation regarding this matter for adult head-injured patients [2]. However, there are no conclusive studies done to provide answers for the paediatric patient. Despite the frequent occurrence of head injury in children, the paediatric literature does not provide us with consistent management guidelines $[4,8,10,13]$. The Evidence-based guidelines for the treatment of severe TBI have not yet been established for children $[8,13]$. The aim of this study is to present our experiences with the unilateral decompressive craniectomy (UDC) and to offer decision help to all neuroemergency healthcare professionals, who deals with such cases.

\section{Material and Methods}

Between 1996 and 2020 we treated 37 children with severe brain injury (GCS of 3 to 8 ) and primary intracranial pressure (ICP) of $30 \mathrm{mmHg}$ or higher. In all cases follow up data could be obtained. Mean age was

Citation: Hejazi N, Al-Kassab M (2021) Management of Severe Traumatic Brain Injury in Children by Decompressive Craniectomy: Technical Note and Therapy Concepts. Neurosurg Cases Rev 4:075. doi. org/10.23937/2643-4474/1710075

Accepted: July 26, 2021; Published: July 28, 2021

Copyright: (C) 2021 Hejazi N, et al. This is an open-access article distributed under the terms of the Creative Commons Attribution License, which permits unrestricted use, distribution, and reproduction in any medium, provided the original author and source are credited. 


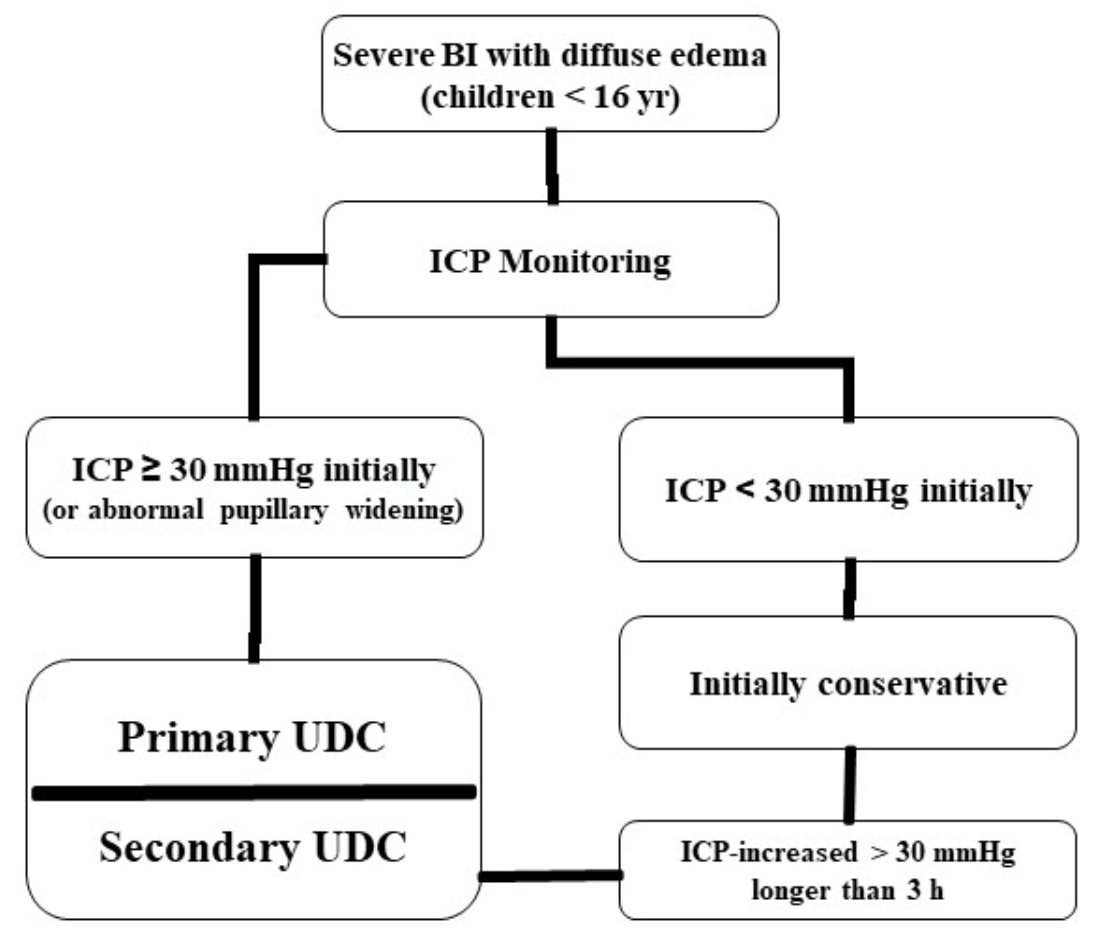

Figure 1: Algorithm of our management strategies of severe brain injury in children.

6.5 years (range 2 years to 16 years). 20 (54\%) of the injured children were male and 17 (46\%) were female. All patients were admitted within 2 hours following their head injury. The mechanism of injury, clinical features at presentation and scan interpretations were recorded for each child.

\section{Management strategy}

Inclusion criteria were the presence of a severe TBI according to a GCS lower than 8 and diffuse brain injury with generalized oedema without significant mass lesion on initial computerized tomography (CT) scans (Figure 1). Patients with large mass lesions were excluded from the study. The time span between the trauma and the operation was between 30 and 75 min. In accordance with our own management regime, initially, an intraparenchymal pressure transducer was routinely inserted into the frontal region to control ICP. If the initial ICP $30 \mathrm{mmHg}$ or higher (especially with acute bilateral abnormal pupillary widening), immediate unilateral decompressive craniectomy (UDC) was performed (Figure 1).

The UDC technique began with a curvilinear incision in the frontotemporal region. The temporal muscle was opened with an inverted anchor incision. Preparation of a myocutaneous flap and craniectomy with elevation of a free frontotemporo basal bone flap was performed. We leave the parietal and occipital regions intact to support the decompressed cerebral hemisphere. The superior sagittal sinus was not exposed to prevent unnecessary blood loss. It is of crucial importance to rongeur the temporal bone down to the level of the middle fossa to decompress the midbrain and the brainstem (Figure 2a, Figure $2 \mathrm{~b}$ and Figure 3 ). The dura mater was opened in a stellate fashion performing an enlarging duraplasty (Figure 3).

The vessels crossing the durotomy margin were protected by carefully shoving of the duraplasty material (synthetic dural substitute neuro-patch) under the dura at the edge of the craniotomy. The major vessels were additionally protected by using TachoComb and a second layer of duraplasty to avoid venous compression at the edge of the craniectomy (Figure 3). The temporal muscle, but not the temporal fascia, was sutured meticulously in a water-tight closure. The galea and skin were closed in standard fashion. The bone was stored under sterile conditions at $-80{ }^{\circ} \mathrm{C}$. Standard treatment protocol for intracranial hypertension consisting of head elevation $\left(30^{\circ} \mathrm{C}\right)$, cerebral perfusion pressure (CPP) monitoring with a CPP minimum of $60 \mathrm{mmHg}$, hyperventilation (paCO ${ }_{2}$ of $32-35 \mathrm{mmHg}$ ), osmotherapy with mannitol was followed. Reimplantation was performed 8 to 12 weeks after surgery.

\section{Results}

Most of the children were injured in falls (50\%) or in motor vehicle accidents (32\%). Child abuse was present in three cases, and was subsequently substantiated by protective services and law-enforcement investigations. All patients had at least 8 months of follow-up evaluation.

The pictures of two children are shown in Figure 4 (case 15 and case 27), 1 month after UDC and before reimplantation of bone flap (left), and 1 year after UDC (right).

Both children have at admission initial ICP of 36 

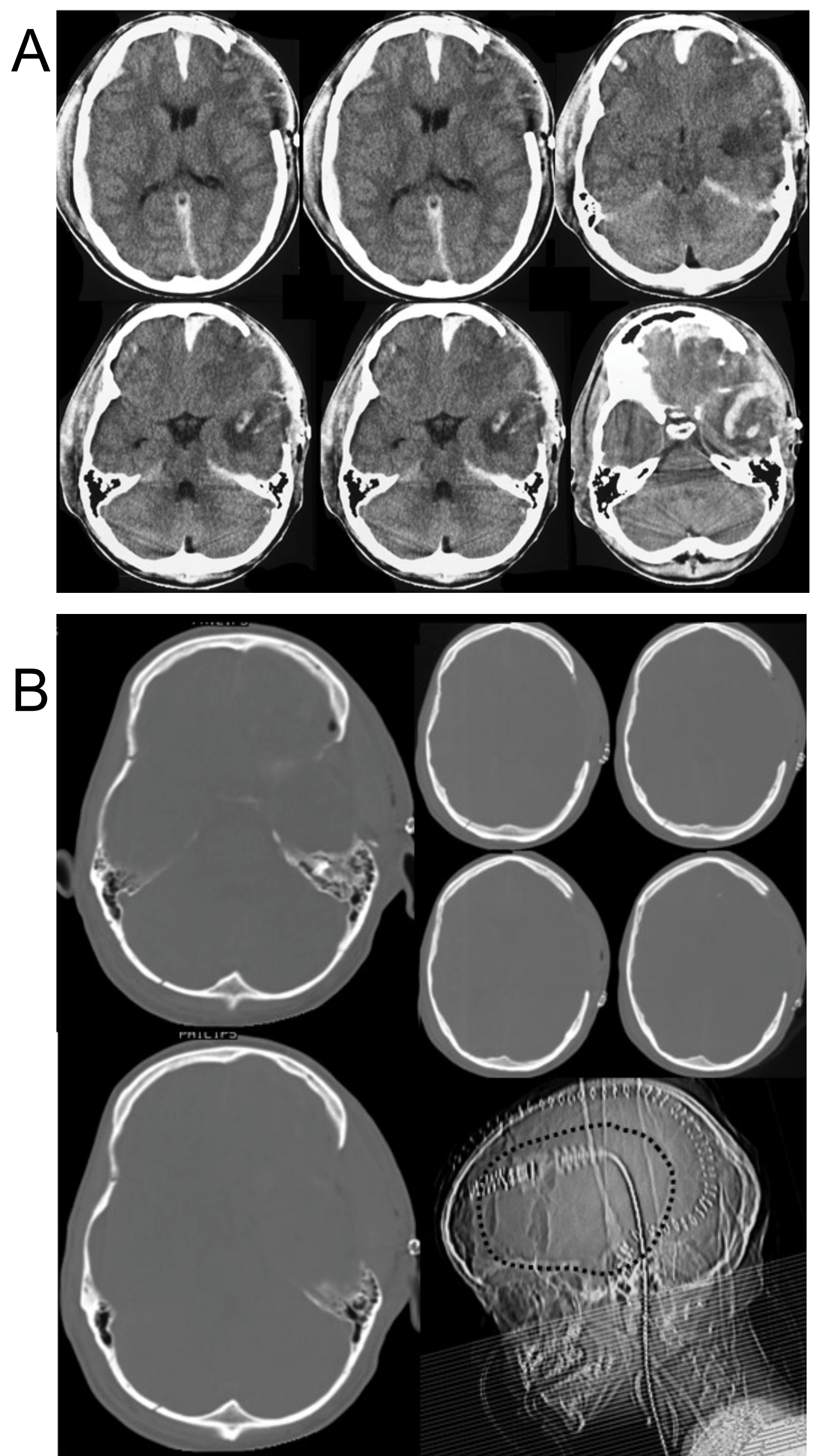


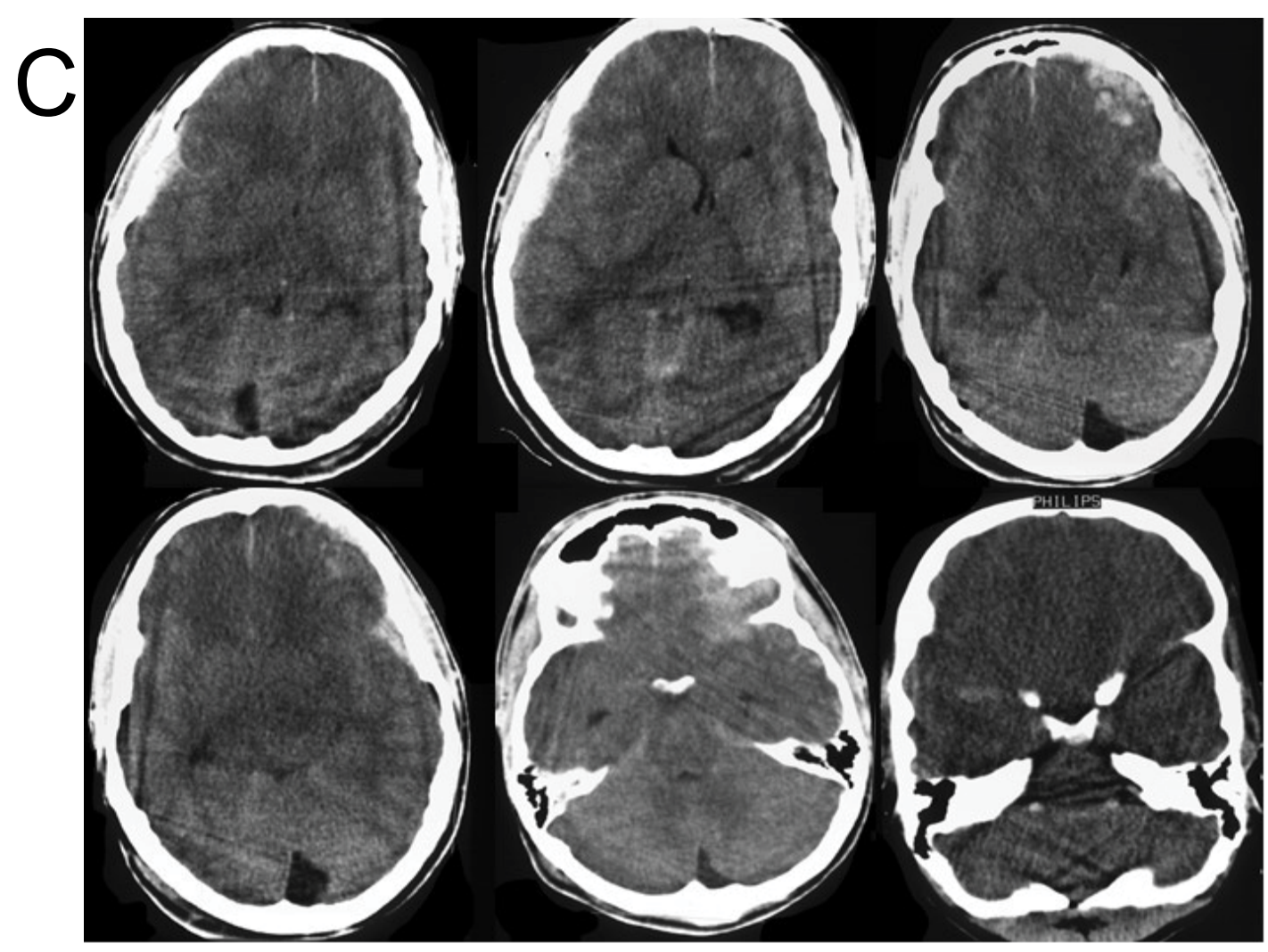

Figure 2a: (a) Admitting CT scans in a 7 years old child with severe brain injury. Note the diffuse brain swelling, the obliterated mesencephalic cisterns, and the presence of small intra-parenchymal hemorrhages left frontal. The initial ICP after inserting of an intraparenchymal pressure transducer was $36 \mathrm{mmHg}$ (case 15); (2b) Early CT scans of the same child after the UDC showing the sufficient decompression of the mesencephalic region. Note the enlargement of the pre-existing contusions left frontally without a significant mass effect; (2c) Early CT scans of the same child after the UDC showing the bone structure and the craniotomy reaching the temporal skull base and decompress the middle brain area (bone technique).
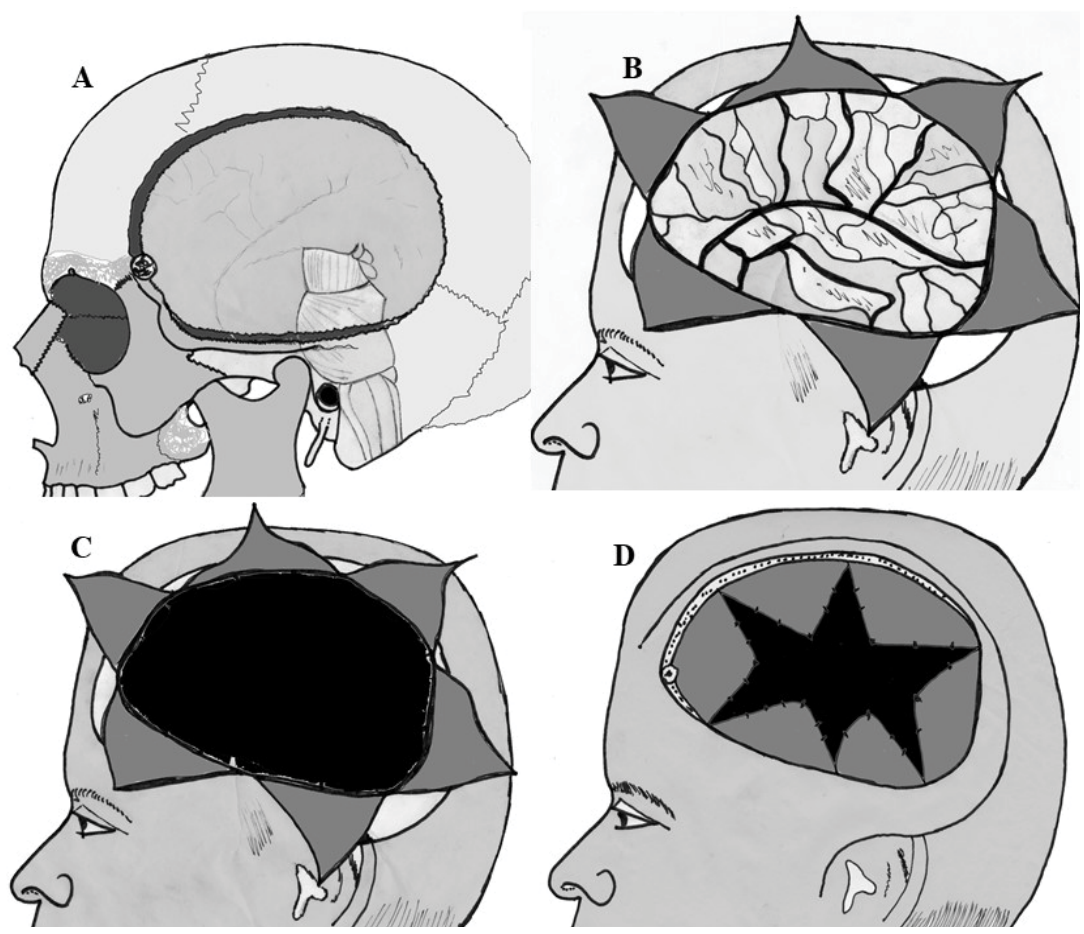

Figure 3: Illustrations of the UDC technique. (A) It is of crucial importance to rongeur the temporal bone down to the level of the middle fossa to decompress the midbrain and the brainstem; $(B, C, D)$ The dura mater was opened in a stellate fashion performing an enlarging duraplasty. The vessels crossing the durotomy margin were protected by carefully shoving of the duraplasty material (synthetic dural substitute neuro-patch, black in $C+D$ ) under the dura at the edge of the craniotomy. The major vessels were additionally protected by using Tacho Comb and a second layer of duraplasty to avoid venous compression at the edge of the craniectomy (black). 


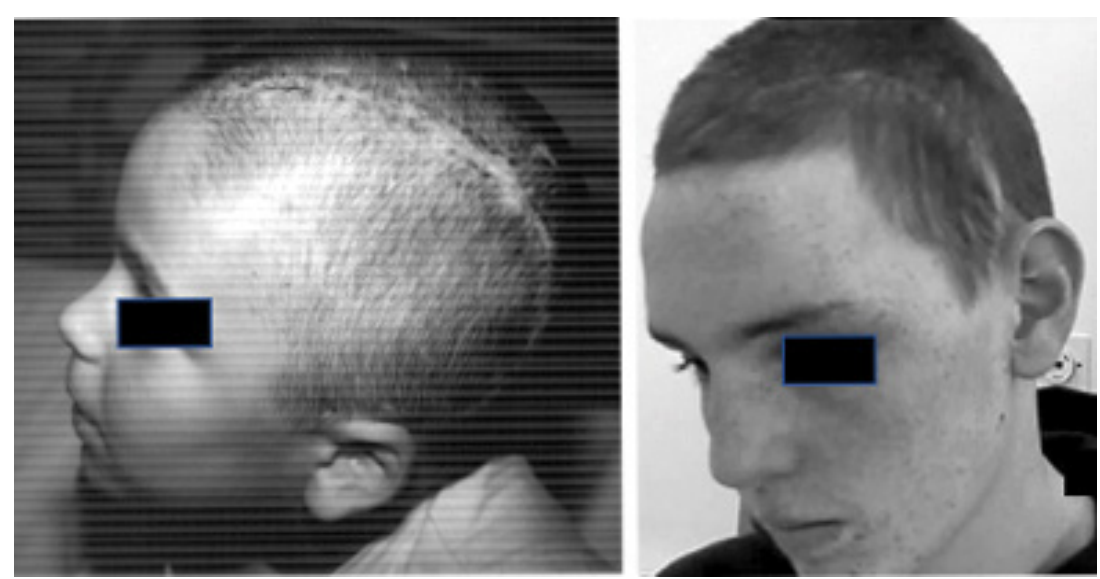

Figure 4: The picture of two children 1 month (left, before the reimplantation of the bone flap, case 15) and 1 year (right, case 27) after UDC, both children have at admission initial ICP of $36 \mathrm{mmHg}$ with herniation signs and compression of the perimesencephalic cisterns on the admitting CT scan.

$\mathrm{mmHg}$. The admitting CT scans (Figure 2c) in case 15 demonstrated the diffuse brain swelling, the obliterated mesencephalic cisterns, and the presence of small intraparenchymal haemorrhages left frontal. The initial ICP after inserting of an intraparenchymal pressure transducer was $36 \mathrm{mmHg}$. The postoperative early CT scans of the same child after the UDC showed sufficient decompression of the mesencephalic region, slightly enlargement of the pre- existing contusions left frontally without a significant mass effect (Figure 2a).

UDC achieved a significant decrease in ICP less than $20 \mathrm{mmHg}$ immediately after urgent surgery. Three patients died during the first $48 \mathrm{~h}$ post emergency operation, all the other (34 cases) are still alive with the following Glasgow Outcome Scores: good recovery (27), moderate disability (5), and vegetative state (2). 2 children remain despite of an acute early UDC postoperatively in vegetative state. These 2 children were 7 and 11 years old and have at admission a GCS less than 8 with bilateral maximal pupillary widening for more than 1 hour.

\section{Discussion}

\section{Appearance of diffuse brain swelling on CT in children}

The most important sign of diffuse brain swelling on CT is compressed or obliterated mesencephalic cisterns, which usually well imaged in healthy children. The presence of small intraparenchymal hemorrhages $<$ $15 \mathrm{~mm}$ ) is usually associated with diffuse axonal injury and has also been shown to be of major predictive importance in the outcome of pediatric patients with diffuse brain swelling [14]. The evaluation of ventricular size represents a problem in the pediatric population. Snoek, et al. warned that, until clear defined criteria for minimum ventricular size in normal children are set, caution is needed when interpreting "small" ventricles $[10,15]$. Our own analysis, as well as that of Teasdale, et al. indicated that lateral ventricular size is not an accurate predictor of raised ICP $[16,17]$. Berger, et al. reported a diffuse brain swelling incidence of $29 \%$ in their series of severely head injured children, who fulfilled these CT criteria $[6,18]$. However, in that report the indicative CT pattern was seen in only $15 \%$ of those patients with GCS scores greater than 8 , while it was present in $41 \%$ of those with lower GCS scores $[8,16,18]$.

\section{Decompressive craniectomy in children}

Initial enthusiasm for the decompressive craniotomy (DC) in the treatment of severe cerebral oedema has been tempered by disappointing results. Venous compression at the edge of the craniectomy could be responsible for an increase of edema and ICP after radical circumferential craniectomy [19-21]. The major vessels crossing the durotomy margin could be protected in our cases by carefully shoving of the duraplasty material under the dura at the edge of the craniotomy. Complications related to the larger DC and some late neurological deficits postoperatively might have been due to the size of the DC. On the other hand, the results in children with head injury treated by DC were more worthwhile if compared with those in adults $[4,7,12]$. Guerra, et al. found, that the mortality rate decreased in patients operated on later than 24 hours in adults and 12 hours in children $[19,20]$. As there are few available data in the pediatric population, many of the treatment modalities used in children with severe TBI have been extrapolated from those described in the adult literature. There are, however, many anatomical and physiological differences that impact on the ability of children to tolerate and respond to injury. Even within the pediatric population, age independently affects the mortality rate in patients with head injury. The Traumatic Coma Data Bank (TCDB) reported post-injury outcome at 6 and 12 months in 103 children who were younger than 15 years of age with severe head injuries [17]. These data show that the outcome at follow up is worse in young patients between the ages of 0 and 4 years and is best in children between the ages of 5 
and 10 years. Cerebral blood flow may be significantly reduced as ICP rises, and cerebral metabolic rate and cerebral oxygen extraction are maximal shortly after injury in children. The smaller subarachnoid space may reduce the buffering capacity for externally applied impact [10]. This means that the brain is very vulnerable to ischemic injury at this stage. Severe early intracranial hypertension may be associated with brain death within a few hours. Hypoxia may be more highly associated with poor outcome in survivors than death $[9,13-$ $15,20]$. Emergency care should be organized to optimize CPP during the golden hour immediately following head injury. The length of time between injury and admission appeared to influence outcome in survivors. Humphreys, et al. found a mortality rate of $39 \%$ in their series of severely head-injured children with diffuse brain swelling. The addition of patients with diffuse brain swelling plus small parenchymal hemorrhages did not appreciably alter these results. A significant observation was that, of the children with diffuse brain swelling who died, $90 \%$ did so within 6 days of the accident [14] The management of raised intracranial pressure (ICP) is the key issue of therapy strategies in severe brain injury. Since the first experience by Cushing in 1905 decompressive craniectomies have been performed for different indications. Most retrospective brain trauma series presented a negative patient selection due to the fact that decompressive craniectomy served as ultimo ratio surgery. In the last decade decompressive surgery became a widely used technique in treating patients after trauma and brain infarction $[7,17]$. Polin, et al. investigated 35 patients who underwent bifrontal craniectomy and correlated them with comparable patients from the Traumatic Coma Data Bank in respect of age, sex, preoperative GCS and maximum ICP [11]. They found both significantly lowered ICP and a favorable outcome in the operated group with best results for pediatric patients ( $44 \%$ good outcome). It is remarkable that 18 out of 35 patients were children $[17,20]$. Taylor, et al. published a first prospective, randomized study on 27 pediatric patients over a period of 7 years and found evidence that decompression may have a good influence on outcome and ICP, but without significant statistical results $[16,22]$. The average craniectomy size was 3 to $4 \mathrm{~cm}$ in diameter without additional dural enlargement. Outcome evaluation was performed after 6 months by a telephone questionnaire [22].

We performed the UDC opening the dura mater in a stellate fashion with an enlarging duraplasty. The vessels crossing the durotomy margin were protected by carefully shoving of the duraplasty under the dura at the edge of the craniotomy. The major vessels were additionally protected by using TachoComb and a second layer of duraplasty to avoid venous compression at the edge of the craniectomy. The temporal muscle, but not the temporal fascia, was sutured meticulously in a water-tight closure [4]. The child dura mater always seems very flexible and stretchable, so that with this kind of dura opening and duraplasty enough capacity was achieved to compensate the space-require of the oedematous brain under the dura. One problem facing the clinician is to determine the length of time that should be allowed for conservative therapy before considering DC. It is widely accepted that DC should not be postponed so long that irreversible brainstem changes occur. However, the exact point of time at which a craniectomy would be most beneficial is unclear. Most patients with massive oedema develop brain herniation within 24 hours after trauma. We consider that in children with primary traumatic cerebral edema with herniation signs, compression of the perimesencephalic cisterns on the admitting CT scan, and initial ICP of $30 \mathrm{mmHg}$ or higher, early UDC not only reduces mortality but also significantly improves outcome. The UDC should be performed immediately before further decompensation occurs, so that additional ischemic insults and irreversible damage of cerebral tissue may be avoided. Because of advanced neurointensive monitoring, the intracranial pressure and cerebral perfusion pressure can be continuously monitored quite easily, and the timing of surgical intervention can be adapted. The good results in children treated with UDC may justify the modification of the recommendations have been made by the Brain Trauma Foundation regarding this matter for headinjured children. We think that the UDC should be considered in selected pediatric patients with severe $\mathrm{BI}$ as immediate emergency modality of treatment, before one misses the important and valuable time to improve outcome.

Decompressive craniectomy entails certain risks for the patient. Intra- and postoperative blood losses in operated children can lower the CPP dramatically. Postoperative complications are further sub- and epidural hemorrhages and an impaired CSF circulation with hydrocephalus and/or subdural hygroma often combined with secondary neurological deficits ("post decompression syndrome"). In order to prevent these complications early defect closure is mandatory. Infections can occur either after decompression or after bone flap replacement. In young children decalcification and lysis of the reimplanted bone flap have been observed (in five cases). Despite these complications, the benefits of the UDC seem to outweigh the disadvantages.

\section{Conclusion}

Our results suggest that in children with primary traumatic cerebral edema with herniation signs, compression of the perimesencephalic cisterns on the admitting CT scan, and initial ICP of $30 \mathrm{mmHg}$ or higher, early UDC not only reduces mortality but also significantly improves outcome. We think that the UDC should be considered in selected pediatric patients with severe $\mathrm{BI}$ 
as immediate emergency modality of treatment, before one misses the important and valuable time to improve outcome. The clinical assessment of head-injured children presents unique difficulties, and therefore agespecific behaviors must be considered.

\section{References}

1. Beattie TF (1997) Minor head injury. Arch Dis Child 77: 8285.

2. Dershewitz RA, Kaye BA, Swisher CN (1983) Treatment of children with posttraumatic transient loss of consciousness. Pediatrics 72: 602-607.

3. Rivara F, Tanaguchi D, Parish RA, Stimac GK, Mueller B (1987) Poor prediction of positive computed tomography scans by clinical criteria in symptomatic pediatric head trauma. Pediatrics 80: 579-584.

4. Kraus JF, Fife D, Cox P, Ramstein K, Conroy C (1986) Incidence, severity and external causes of pediatric brain injury. Am J Dis Child 140: 687-692.

5. Homer CJ, Kleinman L (1999) Technical report. Minor head injury in children. Pediatrics 104.

6. Berger S, Schwarz M, Huth R (2002) Hypertonic saline solution and decompressive craniectomy for treatment of intracranial hypertension in pediatric severe traumatic brain injury. J Trauma 53: 558-563.

7. Gratz R (1979) Accidental injury in childhood: A literature review on pediatric trauma. J Trauma 19: 551-555.

8. Haydel MJ, Preston CA, Trevor JM, Luber S, Blaudeau E, et al. (2000) Indications for computed tomography in patients with minor head injury. NEJM 343: 100-105.

9. Davis RL, Mullen N, Makela M, Taylor JA, Cohen W, et al. (1994) Cranial computed tomography scans in children after minimal head injury with loss of consciousness. Ann Emerg Med 24: 640-645.

10. Snoek JW, Minderhoud JM, Wilmink JT (1984) Delayed deterioration following mild head injury in children. Brain 107: $15-36$.
11. Polin R, Shaffrey M, Bogaev C, Tisdale N, Germanson $\mathrm{T}$, et al. (1997) Decompressive bifrontal craniectomy in the treatment of severe refractory posttraumatic cerebral edema. Neurosurgery 41: 84-92.

12. Jennett B (1996) Epidemiology of head injury. J Neurol Neurosurg Psychiatry 60: 362-369.

13. Singer HS, Freeman JM (1978) Head trauma for the pediatrician. Pediatrics 62: 819-825.

14. Humphreys RP (1991) Complications of pediatric head injury. Pediatr Neurosurg 17: 274-278.

15. Münch E, Horn $P$, Schurer L, Piepgras A, Paul T, et al. (2000) Management of severe traumatic brain injury by decompressive craniectomy. Neurosurgery 47: 315-323.

16. Taylor HG, Yeates KO, Wade SL, Drotar D, Stancin T, et al. (2001) Bidirectional child-family influences on outcomes of traumatic brain injury in children. $\mathrm{J}$ Int Neuropsychol Soc 7: 755-767.

17. Teasdale G, Teasdale E, Hadley D (1992) Computed tomographic and magnetic resonance imaging classification of head injury. J Neurotrauma.

18. The American Association of Neurological Surgeons; The Joint Section on Neurotrauma and Critical Care: Hyperventilation. The Brain Trauma Foundation: 513-520.

19. Hennes H, Lee M, Smith D (1988) Clinical predictors of severe head trauma in children. Am J Dis Child 142: 10451051.

20. Guerra WK, Gaab MR, Dietz H, Mueller JU, Piek J, et al. (1999) Surgical decompression for traumatic brain swelling: Indications and results. J Neurosurg 90: 187-196.

21. Hejazi N, Witzmann A, Fae P (2002) Unilateral decompressive craniectomy for children with severe brain injury. Report of seven cases and review of the relevant literature. Eur J Pediatr 161: 99-104.

22. Mataro M, Poca MA, Sahuquillo J, Pedraza S, Ariza M, et al. (2001) Neuropsychological outcome in relation to the traumatic coma data bank classification of computed tomography imaging. J Neurotrauma 18: 869-879. 